\title{
The Separation of Two Reduction Products of Nitroblue Tetrazolium (Nitro BT) in Paramecium caudatum Using a Microphotometric Image-Analysis System
}

\author{
Kensuke Chikamori, Tsutomu Araki* and Katsuyuki Sato** \\ Department of Chemistry, Naruto University of Education, Takashima, Naruto-cho, Naruto-shi, Tokushima \\ 772-8502, *Department of Systems and Human Science, Graduate School of Engineering Science, Osaka \\ University, Toyonaka, Osaka 560-8531 and ** Department of Biology, Naruto University of \\ Education, Takashima, Naruto-cho, Naruto-shi, Tokushima 772-8502
}

Received for publication September 10, 1997 and in revised form April 27, 1998

\begin{abstract}
Monoformazan (MF) and diformazan (DF) are the reduction products of nitroblue tetrazolium (Nitro BT), commonly used for the histochemical detection of dehydrogenase activity. We designed a microphotometric image-analysis system, and used it to measure the separate amounts of MF and DF in a single Paramecium caudatum. We stained this cell in the presence of succinate and 1methoxy phenazine methosulfate at $30^{\circ} \mathrm{C}$, and took the observed density images (ODI) at $530 \mathrm{~nm}$ and $650 \mathrm{~nm}$ every $3 \mathrm{~min}$ for $15 \mathrm{~min}$. We used the component-separation method to reconstruct the individual absorp-

tion images of MF and DF from a pair of ODIs of the single cell at each reaction time. The estimation of MF and DF quantities in the cell are based on their total absorbance at each reaction time, and molar extinction coefficients at $530 \mathrm{~nm}$ and $650 \mathrm{~nm}$, respectively. Our study indicates the difference in the spatial distribution and also appreciable kinetic differences in the formation between MF and DF, and provides a comparison between the kinetic data from the separate measurements and that taken at $590 \mathrm{~nm}$, which is near the isobestic wavelength of the two formazans $(585 \mathrm{~nm})$.
\end{abstract}

Key words: Nitro BT, Component separation, Image analysis, Paramecium caudatum

\section{Introduction}

Nitroblue tetrazolium (Nitro BT) is commonly used as a final electron acceptor in the histochemical detection of dehydrogenase activity in tissue and cell [16]. Nitro BT reduction produces two reduction products, monoformazan (MF) and diformazan (DF), both of which deposit simultaneously in a specimen. The absorption profiles of DF and MF are different, yet a major part of each overlaps the other $[2,5]$. It is therefore necessary to separate the observed spectrum into the individual component spectra of MF and DF to quantify dehydrogenase activity. One attractive alternative procedure is to measure absorbance at the isobestic wavelength of MF and DF $(585 \mathrm{~nm})$ as described by Butcher [5], and Butcher and Van Noorden [7]. Although this technique is now widely used to convert mean integrated absorbance into a total concentration of the final reduction products of Nitro BT and an absolute unit of enzyme activity [17], only Araki et al. [2]

Correspondence to: Dr. Kensuke Chikamori, Department of Chemistry, Naruto University of Education, Takashima, Narutocho, Naruto-shi, Tokushima 772-8502, Japan. described a direct comparison between the result obtained from in situ separate measurements of MF and DF and that obtained using the isobestic wavelength method in relation to reaction time so far. They succeeded in determining the separate spectral and spatial profiles of individual formazans, which were produced by histochemical succinate dehydrogenase (SDH) reaction in rat liver and skeletal muscle, using multispectral image-analysis [13]. They indicated that the SDH activities, the amount of hydrogen consumed for formation of two formazans, estimated by this technique are comparable to those calculated from measurements at $585 \mathrm{~nm}$ in the different zones of rat liver acinus; however, this study did not try to trace separately the formation of the two Nitro BT-derived formazans in relation to reaction time, because this technique required a large-scale computer to analyze the collected spectral data. In 1991, we developed a new microphotometer system for routine laboratory use, based on multispectral image-analysis [9], and have used this system to measure SDH activity in Paramecium caudatum, a ciliated protozoa, with Nitro BT at near the isobetic wavelength of its formazans $(590 \mathrm{~nm})$ [10].

The aim of this work was to reveal the in situ kinetics 
of individual MF and DF formation from Nitro BT in a single Paramecium cell in the presence of succinate (substrate of succinate dehydrogenase) and 1-methoxy phenazine methosulfate (1-methoxy PMS; an electron carrier), to compare the results with those obtained from the measurement taken at near the isobestic wavelength of Nitro BT formazan $(590 \mathrm{~nm})$, and to indicate the reliability of our designed microphotometer system for the quantitative histochemical research of Paramecium SDH activity with Nitro BT.

\section{Materials and Methods}

\section{Preparation of specimen and measurement}

Details in the preparation of specimen and measurement have been described elsewhere [10].

Paramecium caudatum cells, cultured in a boiled extract of dried lettuce with Klebsiella pneumoniae at $25^{\circ} \mathrm{C}$, were washed once in ice-cold Dryl's physiological solution (pH 7.3), a specified salt solution for experiments with Paramecium cells [12], by centrifugation at $760 \times g$ for $3 \mathrm{~min}$ at room temperature. Finally, precipitated cells were suspended in a small volume of Dryl's physiological solution (0.1-0.2 ml), and immobilized on the surface of a coverslip by centrifugal force at $210 \times g$ for $5 \mathrm{~min}$.

Instrumentation of the microphotometer system was reported previously [9]. Using a flow-cell cuvette to detect SDH activity, we traced the formation of the reduction products of Nitro BT in a single Paramecium cell at specific reaction times. This flow-cell cuvette consisted of a glass slide, a pair of spacers, and a cover slip with immobilized cells. Temperature was controlled at $30^{\circ} \mathrm{C}$ by a thermo-regulated heating plate (Micro Warm Plate DC-MP 100 M, Kitazato, Shizuoka, Japan) placed between a flow-cell cuvette and a microscope stage. The reaction mixture consisted of $100 \mathrm{mM}$ phosphate buffer (pH 7.6), $2 \mathrm{mM}$ Nitro BT (Dotite, Kumamoto, Japan), $1 \mathrm{mM}$ sodium azide (Wako Pure Chemical Industries, Osaka, Japan), $0.1 \mathrm{mM}$ 1-methoxy PMS (Dotite), $50 \mathrm{mM}$ sodium succinate (Wako), and 18\% (w/v) polyvinyl alcohol (PVA; average molecular weight 30,000-70,000; Sigma, St. Louis, MO, USA). PVA was used as a tissue protectant according to Van Noorden and Vogels [15]. After preliminary warming of both the flow-cell cuvette and the reaction mixture at $30^{\circ} \mathrm{C}$ for $5 \mathrm{~min}$, we selected a single Paramecium cell from within the measuring field. The reaction was started by injecting the reaction mixture into a flow-cell cuvette; absorption signals (observed density image; ODI) were then detected under a transmission microscope, using a $20 \times$ objective lens. We defined the specific moment a reaction mixture had covered the selected cell, and its image through the reaction mixture had been adjusted on a monitor, the 0 -min reaction time. ODIs were then taken every three min for $15 \mathrm{~min}$, using the alternative trans-illumination monochromatic beams of $530 \mathrm{~nm}$ and $650 \mathrm{~nm}$. The monochromatic light was obtained through the interference filters (band width $12 \mathrm{~nm}$ FWHM), and the time lapse for the change of wavelength was within $2 \mathrm{sec}$. ODIs were detected with a CCD camera (CS3430, Tokyo Electronic Industry, Tokyo, Japan), and digitized in a frame grabber (Image Mate-N, Nippon Avionics, Tokyo, Japan). The measuring actions were controlled by a computer (PC 9801-VX, NEC, Tokyo, Japan).

\section{Image analysis}

Fig. 1 is a schematic depiction of the principles of image processing. To efficiently erase background

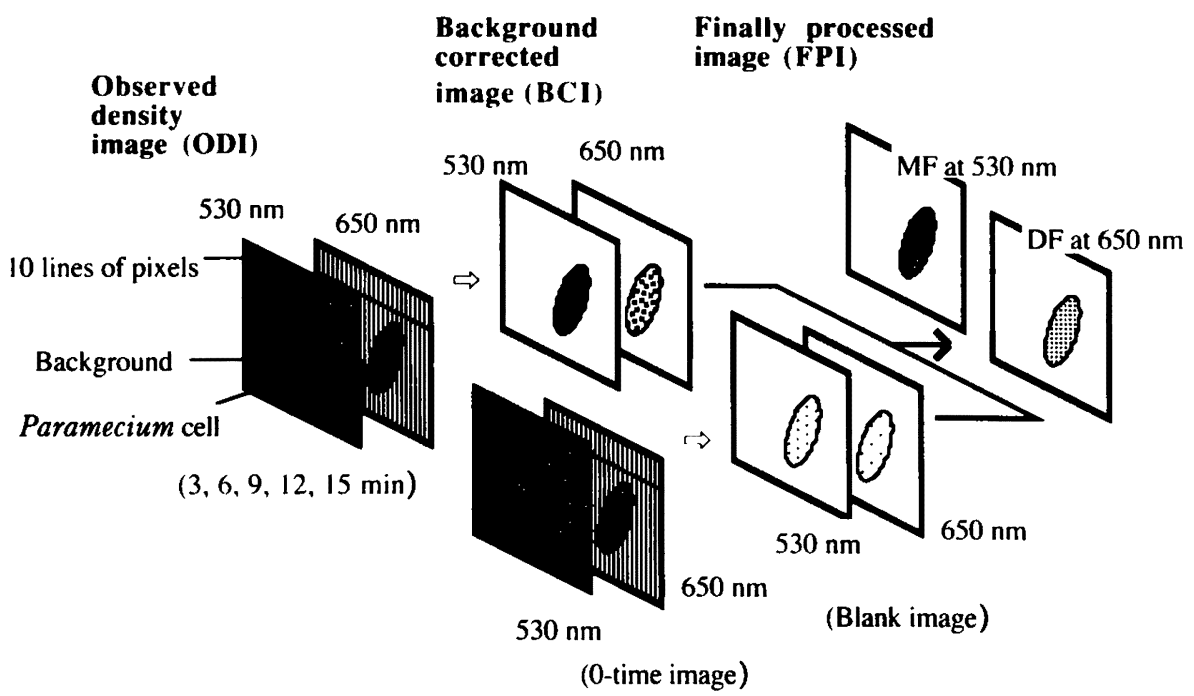

Fig. 1. Schematic representation of image processing. ODIs taken at $530 \mathrm{~nm}$ and $650 \mathrm{~nm}$ in each reaction time. Each ODI was converted to its BCI by taking the average on the absorbance of 10 lines of pixels in an upper part of the background in each ODI. Using the BCI of 0-time image as a blank image, we reconstructed the FPIs for two fromazans from BCIs, using the component-separation method. Square image consists of $256 \times 256$ pixels with 8-bit density, and corresponds to the microscopic measuring field $(242 \mu \mathrm{m} \times 242 \mu \mathrm{m})$. 
absorbance, an average absorbance (AA) was taken on 10 lines of pixels $(10 \times 256$ pixels $)$ at the upper part of the background in each ODI. Taking this AA value as representative of background absorbance in the ODI, the ODI was converted to a background corrected image (BCI). Based on the component spectral profiles of MF and DF [2], absorption images of MF and DF were reconstructed from a pair of BCIs at $530 \mathrm{~nm}$ and $650 \mathrm{~nm}$ at each reaction time by component-separation method. In this processing, the $\mathrm{BCI}$ at $0 \mathrm{~min}$ of reaction time (the 0 -time image) was used as a blank image at each wavelength to correct the contribution of cell-component absorbance to cell image in the BCI. The final resultant processed images (FPI) represent the absorption image of $\mathrm{MF}$ and $\mathrm{DF}$ in a single cell at $530 \mathrm{~nm}$ and $650 \mathrm{~nm}$, respectively. The principle of component separation has been described previously [9].

The absorbance of the FPI was expressed as the total absorbance (TA) at $530 \mathrm{~nm}$ or $650 \mathrm{~nm}$ for each formazan, and was determined as the product of the number of pixels being assigned to a less-than-254 numerical value in the grey scale (corresponding to more than 0.0017 in absorbance) ( $\left.\mathrm{N}_{\text {non-0 }}\right)$ and the average absorbance value of these pixels in a FPI ( $\mathrm{A}_{\text {average }}$ ). Estimation of absorbance of a pixel is described elsewhere [10].

To compare the results obtained from the component separation method with those from near isobestic wavelength measurement, ODIs were taken at $590 \mathrm{~nm}$. Detection of ODI and estimation of TA from the FPI were carried out in the same manner mentioned above.

The commercially available application software (Delta graph 3.5; Nippon Polaroid, Tokyo, Japan) was used to fit the experimental values in an equation, as necessary.

\section{Estimation of the amount of hydrogen $\left(\mathrm{H}_{2}\right)$}

The amount of $\mathrm{H}_{2}$ in mol/cell was calculated from the amounts of MF and DF by equation 1 [5].

Amount of hydrogen $\left(\mathrm{H}_{2}\right)(\mathrm{mol} / \mathrm{cell})=$ [Amount of MF (mol/cell) $]+2 \times[$ Amount of DF (mol/cell)]

On average, the concentration of MF or DF in a cell was calculated by equation 2 .

$$
\mathrm{MF} \text { or } \mathrm{DF}\left(\mathrm{mol} / \mathrm{cm}^{3}\right)=\frac{A_{\text {average }}}{\varepsilon_{\mathrm{MF} \text { or } \mathrm{DF}} \times \mathrm{d} \times 10^{3}}
$$

where $d$ is the average thickness of the Paramecium cell in $\mathrm{cm}$, and $\varepsilon_{\mathrm{MF}}$ and $\varepsilon_{\mathrm{DF}}$ are molar extinction coefficients of MF at $530 \mathrm{~nm}\left(22,100 \mathrm{~mol}^{-1} \mathrm{lcm}^{-1}\right)$ and DF at $650 \mathrm{~nm}$ $\left(29,760 \mathrm{~mol}^{-1} 1 \mathrm{~cm}^{-1}\right)$, respectively. These values are based on the spectral profiles and molar extinction values for two formazans reported by Araki et al. [2] and Butcher [5]. The volume per one pixel (V) in $\mathrm{cm}^{3}$ is given by equation 3.

$$
\mathrm{V}\left(\mathrm{cm}^{3}\right)=\frac{5.8564 \times 10^{-4} \times \mathrm{d}}{65536}
$$

where $5.8564 \times 10^{-4}$ is the area of the microscope-measuring field in $\mathrm{cm}^{2}$ and 65536 is its corresponding pixel num- ber. The total deposited volume of MF or DF in a single Paramecium cell $\left(\mathrm{V}_{\text {deposited }}\right)$ in $\mathrm{cm}^{3}$ is given by equation 4 , and the total amounts of MF or DF are calculated by equation 5 .

$$
\mathrm{V}_{\text {deposited }}\left(\mathrm{cm}^{3}\right)=\mathrm{V}\left(\mathrm{cm}^{3}\right) \times \mathrm{N}_{\text {non- }-0}=\frac{5.8564 \times 10^{-4} \times \mathrm{d} \times \mathrm{N}_{\text {non-0 }}}{65536}
$$

Total amount of MF or DF (mol/cell)

$$
\begin{aligned}
& =(\text { concentration of } \mathrm{MF} \text { or } \mathrm{DF}) \times \mathrm{V}_{\text {deposited }}
\end{aligned}
$$

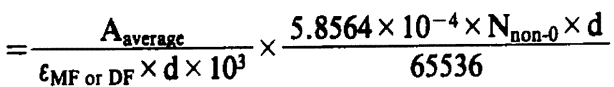

$$
\begin{aligned}
& =\frac{A_{\text {average }} \times N_{\text {non- } 0} \times 5.8564 \times 10^{-7}}{\varepsilon_{\text {MF or DF }} \times 65536} \\
& =\frac{\mathrm{TA} \times 5.8564 \times 10^{-7}}{\varepsilon_{\mathrm{MF} \text { or DF }} \times 65536}
\end{aligned}
$$

\section{Results}

\section{Processed image and spatial distribution pattern of $M F$} and $D F$

To indicate changes in the images through the processing and reaction-time steps, photographs of images in Figs. 2 and 3 were taken directly from the measurement-system monitor. Background-correction results in a decrease in background density with a concomitant decrease in cell-image density in BCIs (Fig. 2B, E), but enhances the contrast between cell-image and its background.

In FPI, the regular striped pattern of formazan deposition is seen in the periphery of the Paramecium cytoplasm (Fig. 2C). This pattern can not be revealed until the BCI has been processed by component-separation. The distribution of MF is rather homogeneous, but its density is somewhat higher in the cytoplasm surrounding the macronucleus. DF, which deposits heterogeneously, exhibits high density in the cytoplasm to the right of the macronucleus.

The area of formazan deposition and its density increase concurrently with reaction time for both MF and DF FPIs (Fig. 3). In the first 3 min of reaction time, only MF is deposited, mainly at the upper right region of the macronucleus (Fig. 3A). This same region shows a great increase in DF density after $6 \mathrm{~min}$ of reaction time (Fig. 3G). The differences in spatial distribution between the two formazans and the regularly striped pattern become more distinct in the FPIs at the longer reaction time (Fig. $3 \mathrm{E}, \mathrm{J})$.

\section{Kinetics of MF and DF formation}

To clarify the progressive formation of MF and DF as represented in the images in Fig. 3, we estimated the TA values in the FPIs for both formazans at each reaction time. Amounts of the two formazans were then calculated from TA values, using equation 5. These values are summarized in Table 1 , and include values of $\mathbf{N}_{\text {non-0 }}$ and $A_{\text {average. }}$ In $\mathrm{MF}$ formation, both values are nearly constant after $12 \mathrm{~min}$ in $\mathrm{N}_{\text {non-0 }}$, and after $9 \mathrm{~min}$ in $A_{\text {average }}$. DF for- 


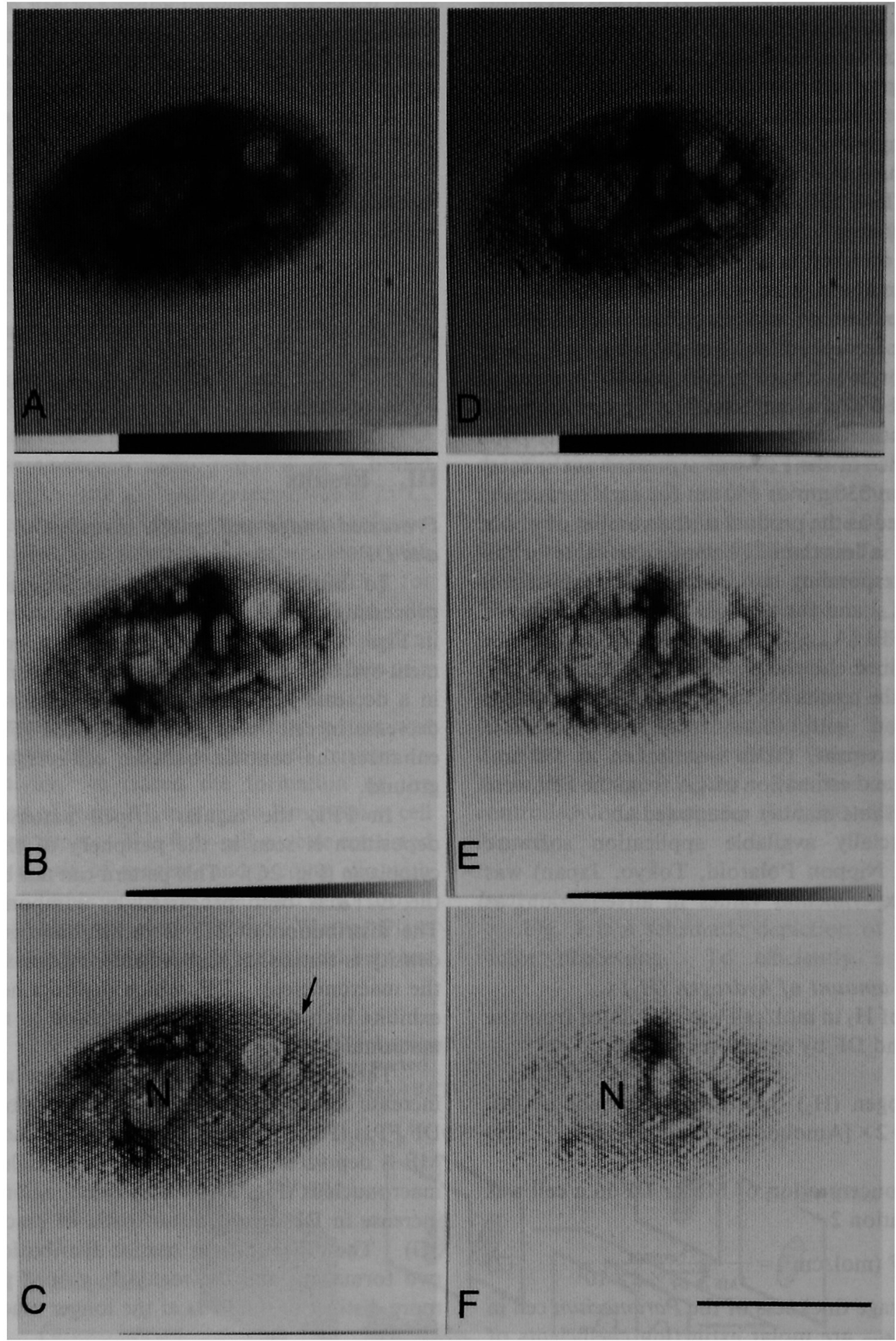

Fig. 2. Images at each step of processing in the component-separation method. (A) ODI at $530 \mathrm{~nm}$; (B) BCI of image A; (C) FPI for MF at $530 \mathrm{~nm}$; (D) ODI at $650 \mathrm{~nm}$; (E) BCI of image D; (F) FPI for DF at $650 \mathrm{~nm}$. Reaction time: 9 min. Arrow indicates regular striped pattern in the periphery of the Paramecium cell. Scale bar $=100 \mu \mathrm{m}$. Gray scale is indicated under the lower edge of each photograph. N, Macronucleus. These photographs were taken directly from the monitor screen of measurement system.

Fig. 3. Finally processed images (FPIs) from each reaction time. A-E: FPI for MF at $530 \mathrm{~nm}$; Reaction time: (A) $3 \mathrm{~min},(\mathbf{B}) 6 \mathrm{~min}$, (C) $9 \mathrm{~min}$, (D) $12 \mathrm{~min}$, (E) $15 \mathrm{~min}$. F-J: FPI for DF at $650 \mathrm{~nm}$; Reaction time: (F) $3 \mathrm{~min}$, (G) $6 \mathrm{~min}$, (H) $9 \mathrm{~min}$, (I) $12 \mathrm{~min}$, (J) $15 \mathrm{~min}$. Arrows indicate regular striped pattern in periphery of the Paramecium cell. Scale bar $=100 \mu \mathrm{m}$. These photographs were taken directly from the monitor screen of measurement system. 


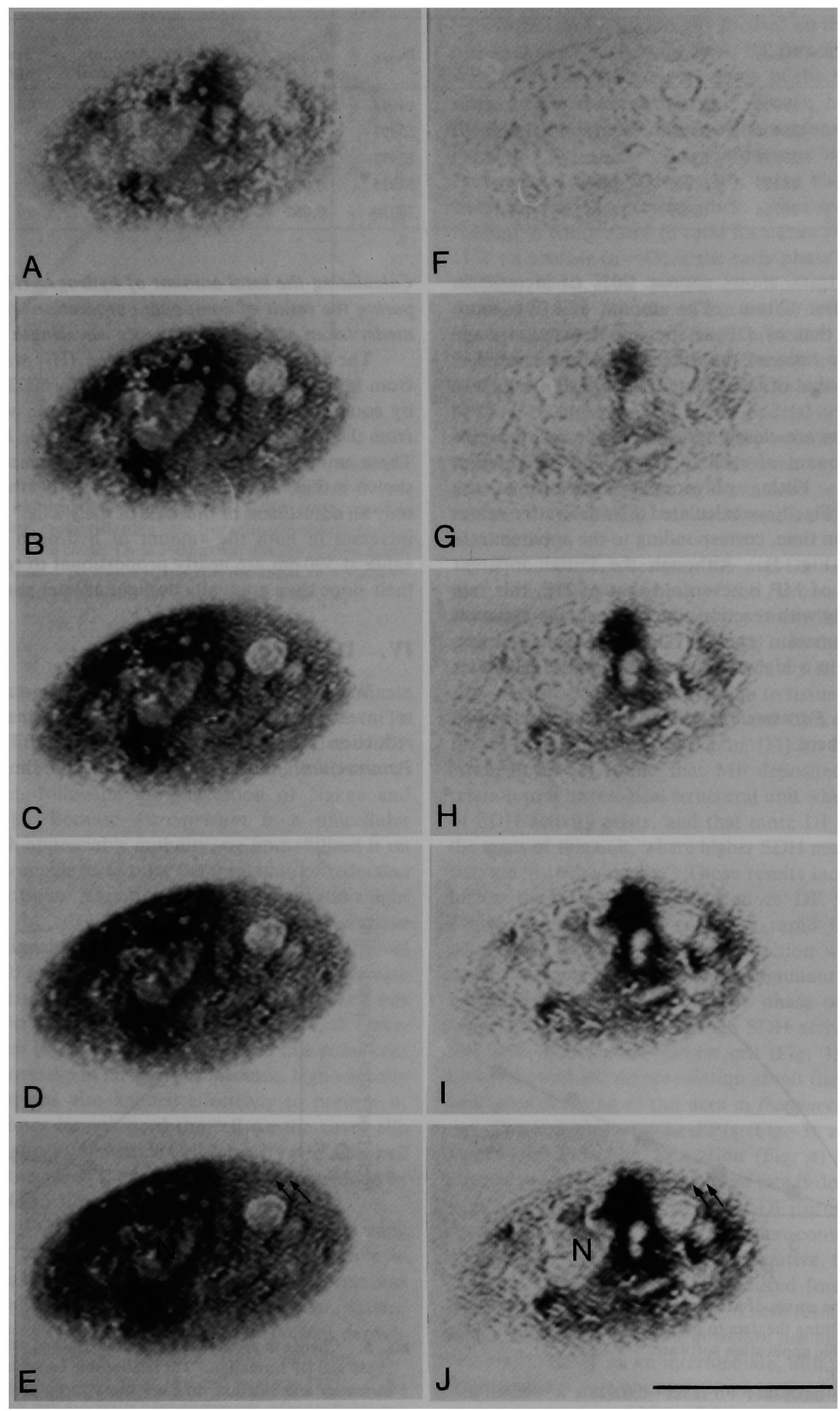

Fig. 3 
Table 1. Values estimated from FPI and the amount of hydrogen

\begin{tabular}{cccccccccc}
\hline $\begin{array}{c}\text { Reaction time } \\
\text { (min) }\end{array}$ & $\mathrm{N}_{\text {non-0 }}$ & $\mathbf{A}_{\text {average }}$ & $\mathrm{MF}$ & $\begin{array}{c}\text { Amount } \\
\text { (pmol/cell) }\end{array}$ & $\mathrm{N}_{\text {non-0 }}$ & A $_{\text {average }}$ & TF & $\begin{array}{c}\text { Amount } \\
\text { (pmol/cell) }\end{array}$ & $\begin{array}{c}\text { Total hydrogen } \\
\left(\mathrm{pmol} \mathrm{H}_{2} / \text { cell) }\right.\end{array}$ \\
\hline 3 & 19006 & 0.056 & 1064 & 0.43 & 14698 & 0.019 & 279 & 0.09 & 0.61 \\
6 & 25404 & 0.080 & 2032 & 0.82 & 20997 & 0.026 & 545 & 0.17 & 1.16 \\
9 & 25899 & 0.095 & 2460 & 0.99 & 22957 & 0.041 & 941 & 0.30 & 1.59 \\
12 & 28325 & 0.095 & 2690 & 1.09 & 28025 & 0.051 & 1429 & 0.46 & 2.01 \\
15 & 28736 & 0.099 & 2844 & 1.15 & 28930 & 0.062 & 1793 & 0.57 & 2.29 \\
\hline
\end{tabular}

mation, in contrast, shows approx. $20 \%$ of increase in

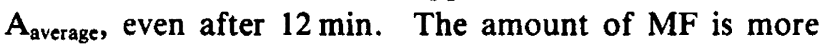
than 40 times that of DF at the initial reaction stage ( $3 \mathrm{~min}$ ), but the ratio of the difference is much smaller, approx. 2 times that of DF, at later reaction stages (12 and $15 \mathrm{~min}$ ).

These ratios are clearly reflected in the relationships between the amount of each formazan and the reaction time (Fig. 4). Fitting polynomial equations to the relationships in Fig. 4, we calculated their derivative values for each reaction time, corresponding to the apparent rate of formation (Fig. 5). Although the initial apparent formation rate of $\mathrm{MF}$ is sevenfold that of $\mathrm{DF}$, this rate decreases steeply with reaction time; in fact, the apparent rate of DF formation gradually increases with reaction time, resulting in a higher value of DF than of MF after $12 \mathrm{~min}$.

One single Paramecium cell was used to obtain all data described here.

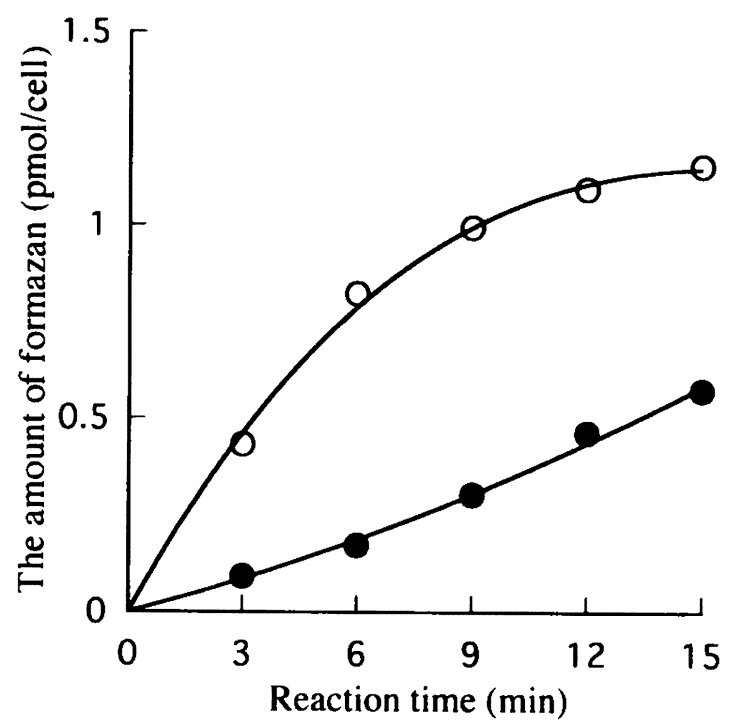

Fig. 4. Formation curves of MF and DF with reaction time. Lines were drawn by fitting the data to polynomial equations using commercially available application software.

MF (open circle):

$\mathrm{y}=0.0001543 \mathrm{x}^{3}-0.009444 \mathrm{x}^{2}+0.1838 \mathrm{x}-0.009287 ; \mathrm{R}^{2}=0.998 ; \mathrm{p}<$ 0.01 .

DF (solid circle): $y=0.0008333 x^{2}+0.02564 x-0.02142 ; R^{2}=0.996$;
Calculating the total amount of hydrogen $\left(\mathrm{H}_{2}\right)$ and comparing the result of component separation with measurements taken at near the isobestic wavelength $(590 \mathrm{~nm})$

The total amount of hydrogen $\left(\mathrm{H}_{2}\right)$ was calculated from individual amounts of the two formazans (Table 1) by equation 1 . The TA value at $590 \mathrm{~nm}$ was obtained from the measurement of another one single Paramecium. These amounts were plotted against the reaction time, as shown in Fig. 6. Both curves fit well together, requiring only an adjustment of the scale of the y-axis. Up to $6 \mathrm{~min}$, increases in both the amount of hydrogen and the TA value at $590 \mathrm{~nm}$ are nearly proportional to reaction time; their slope then gradually declines at later reaction stages.

\section{Discussion}

We used our microphotometric image-analysis system to investigate in situ kinetics in the formation of two reduction products derived from Nitro BT of a single Paramecium. This cell was stained in the presence of

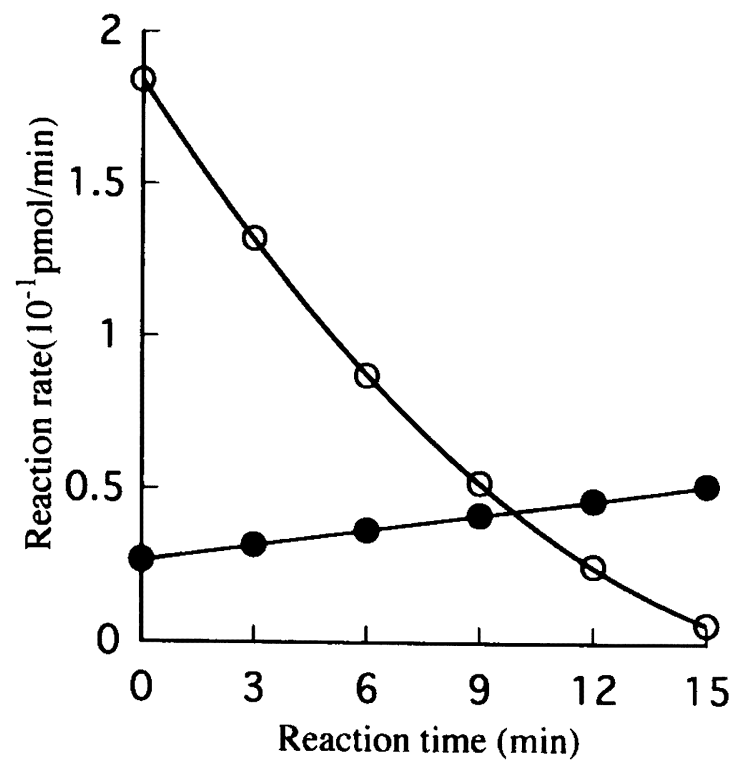

Fig. 5. Change in apparent reaction rate tracked with reaction time in MF and DF formation. The relationship between the amounts of formazan and reaction time are fitted to polynomial equations. Derivative values for MF (open circle) and DF (solid circle) were plotted against reaction time. 


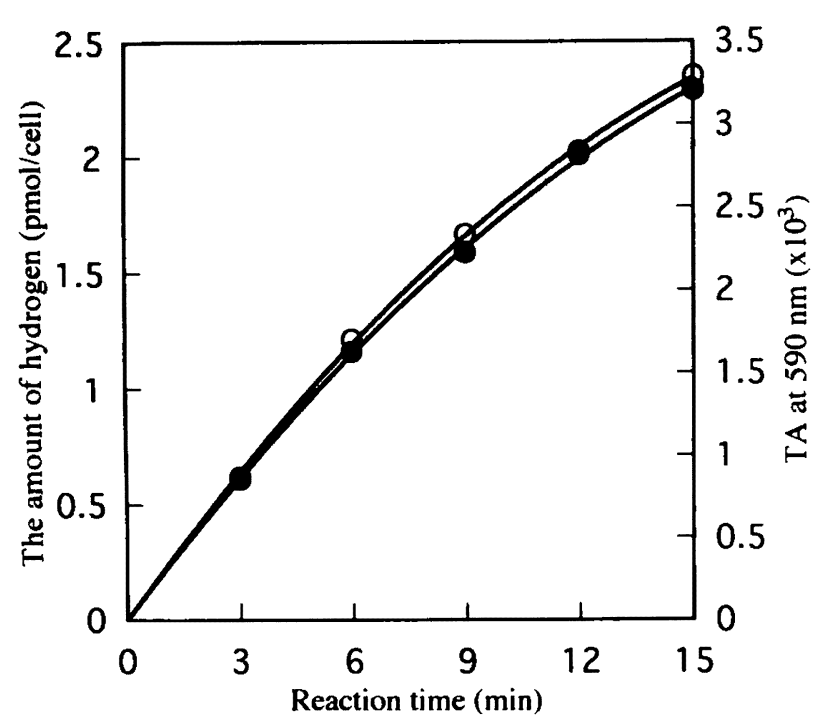

Fig. 6. Comparison between kinetic data estimated from component separation and that obtained from the measurement at $590 \mathrm{~nm}$, near isobetic wavelength of two formazans. Open circle: Amount of hydrogen was estimated using data from the component-separation method. Solid circle: TA at $590 \mathrm{~nm}$ was directly plotted againt the reaction time. To directly compare the shape of formazanformation curves, we adjusted the length of the y-axis.

succinate and 1-methoxy PMS as the substrate for succinate dehydrogenase and an electron carrier, respectively. To avoid any statistical error in studying the formation of MF and DF, we traced the density image of a single cell at each specified time, following the suggestion of Nakae and Stoward [14]. Because Paramecium is a unicellular organism with appreciable mobility, we immobilized it on a cover slip to enable us to trace the formation of reduction products over time. Varying cellular thickness and a light scattering at the edge of the sample combine to cause unfavorable signal fluctuations, and this was an additional impediment to progress. Not only to immobilize but also to create a uniform cell thickness while the selected cell was in the measuring field, we applied a centrifugal force, following Barer [4]. Although we observed that some cells were off the coverslip in an aqueous medium, high-viscosity PVA medium was also applied effectively to prevent it. These procedures immobilized the cell on the cover slip surface during measurement. Cell integrity was also well preserved by the use of PVA medium, as recommended by Van Noorden and Vogels [15].

Processing ODIs by background correction, using 0-time image as a blank image, was quite effective in reconstructing the individual FPIs for the two formazans from ODIs via BCIs. This clearly revealed regular, striated patterns. Paramecium mitochondria are present densely around the kinetosome of cilia, food vacuoles, and contractile vacuoles, and near the cell surface [18]. Cilia cover the entire cell body in arrangements of parallel rows [19]. The regular, striated patterns in the FPIs (Figs. 2,
3) represent rows of Paramecium mitochondria.

In this study, we initially focused on testing the ability of our system to separate Nitro BT formazans; therefore, we did not measure the specimen in the absence of substrate, and do not have that data. We can, however, estimate the contribution of non-specific formazan formation, based on our study of an initial rate measurement of Paramecium SDH activity [10], using the same staining conditions as the present work. Non-specific-formazan formation contributed to total formazan formation about $11 \%$ on average $(n=43)$ in the early phase (up to $3 \mathrm{~min}$ of reaction time), and subsequent, non-specific formazan formation was not linear but rather constant. These results indicate that the results presented here include a higher ratio of non-specifically formed formazans during the initial phase (up to $3 \mathrm{~min}$ ) than in subsequent phases. FPIs representing typical mitochondrial arrangement in a Paramecium cell, together with the estimated extent of the contribution of non-specific formazan to total formazan formation indicate that at least close to $90 \%$ of total formazan formation is attributable to Paramecium SDH activity.

The difference between MF and DF formation in the spatial-distribution pattern of the Paramecium is consistent with results of SDH activity in rat liver and skeletal muscle reported by Araki et al. [2]. In these tissues, heterogeneous distribution of SDH activity has been detected enzyme histochemically in relation to tissue architecture as reported by Anderson et al. [1] and Chikamori et al. [8] for rat liver, and Chikamori et al. [11] for skeletal muscle. Araki et al. [2] found that MF deposited uniformly in relation to a histological structural unit where the gradient of SDH activity exists, and that more DF is produced in the areas of this unit, where higher SDH activity is detected enzyme histochemically. Those results indicated that the higher the SDH activity, the more DF was produced. Thus, our result which shows a rapid increase in DF production at a later phase of reaction was confined to nearly the same area in which predominant MF formation had already occurred in a early phase of reaction and suggests that a particularly high SDH activity localizes in this area in this Paramecium cell (Fig. 3). At present, however, we have no speculation about the structural and biological meaning of this area in Paramecium.

These changes in the FPIs (Fig. 3) and also in the kinetics of formazan formation (Fig. 4) show that MF initially predominates, its apparent rate is decreasing steeply with the concomitant increase in DF formation (Fig. 5). Auclair and Voisin [3] described that complete reduction of Nitro BT to formazan is a consecutive, two-step procedure involving a two-electron-reduced form of Nitro BT (MF) as a stable intermediate. These results indicate that formazan formation in Paramecium has also proceeded in two steps via MF as an intermediate, although there is no comparable report about in situ kinetics in the formation of two formazans of Nitro BT in terms of the histochemical detection of dehydrogenase activity. That MF forma- 
tion apparently ceases toward the later reaction stages (Figs. 4,5 ) suggests that MF is consumed for DF formation, which occurs at a rate nearly equal to that of MF formation. This kinetic behavior is similar to that of Butcher and Altman [6], who describe the separate measurement of two formazans derived from neotetrazolium, using an extraction method; formazan formations from ditetrazolium salts may be similar, regardless of minor differences in their chemical structures.

As mentioned earlier, Araki et al. [2] have already shown that the amount of hydrogen estimated by multispectral image analysis is comparable to that obtained by isobestic wavelength measurement. In the preasent work, we have shown that the kinetic data obtained from the component-separation method that used ourown designed microphotometric image-analysis system are comparable to those obtained from near isobetic wavelength measurement only by adjustment of the y-axis scale. This means that the increase in TA value at $590 \mathrm{~nm}$ reflects faithfully the kinetics of the increase in amount of hydrogen, consumed for Nitro BT-reduction. Thus, near isobestic wavelength measurement at $590 \mathrm{~nm}$ can be used to quantify the SDH activity in Paramecium.

\section{Acknowledgments}

We gratefully acknowledge the technical contribution of Kei Horie, Department of Mechanical Engineering, University of Tokushima (present address: Nikon Co., Tokyo, Japan) to this work.

This work was partly supported by Grant-in-Aid No. 0987570 from the Japan Ministry of Education.

\section{References}

1. Anderson, B., Zierz, S. and Jungermann, K.: Alteration in zonation of succinate dehydrogenase, phosphoenolpyruvate carboxykinase and glucose-6-phosphatase in regeneration rat liver. Histochemistry 80; 97-101, 1984.

2. Araki, T., Chikamori, K., Sasaki, K., Minami, S. and Yamada, M.: Topographic estimation by component spectroanalysis of two formazans of nitroblue tetrazolium in tissue section. Histochemistry 86; 567-572, 1987.

3. Auclair, C. and Voisin, E.: Nitroblue tetrazolium reduction. In "CRC Handbook of Methods for Oxygen Radical Research" ed. by R. A. Greenwald, CRC Press Inc., West Palm Beach, 1985, pp. 123-131.

4. Barer, M. R.: New possibilities for bacterial chemistry cytoche- mistry: Light microscopical demonstration of $\beta$-galactosidase in unfixed immobilized bacteria. Histochem. J. 23; 529-533, 1991.

5. Butcher, R.G.: The measurement in tissue sections of two formazans derived from nitroblue tetrazolium in dehydrogenase reactions. Histochem. J. 10; 739-744, 1978.

6. Butcher, R. G. and Altman, F.P.: Studies on the reduction products of tetrazolium salts. II. The measurement of the half reduced and fully reduced formazans of neotetrazolium chloride in tissue sections. Histochemie 37; 351-363, 1973.

7. Butcher, R. G. and Van Noorden, C. J. F.: Reaction rate studies of glucose-6-phosphate dehydrogenase activity in sections of rat liver using four tetrazolium salts. Histochem. J. 17; 993$1008,1985$.

8. Chikamori, K., Araki, T. and Yamada, M.: Pattern analysis of heterogenous distribution of succinate dehydrogenase in single rat hepatic lobule. Cell. Mol. Biol. 31; 217-222, 1985.

9. Chikamori, K., Yamamoto, A. and Araki, T.: A microphotometer for multispectral image analysis. Instrumentation and application on multicolor-stained specimen. Acta Histochem. Cytochem. 24; 395-403, 1991.

10. Chikamori, K., Araki, T. and Sato, K.: Succinate dehydrogenase (SDH) activity in single Paramecium caudatum cells. Acta histochem. 100; 25-36, 1998.

11. Chikamori, K., Shimizu, T., Shinohara, T., Fukui, K., Araki, T. and Yamada, M.: Cytophotometry for succinate dehydrogenase activity in relation to tissue protein content with tetranitro blue tetrazolium (TNBT). Acta Histochem. Cytochem. 19; 241-251, 1986.

12. Dryl, S.: Antigenic transformation in Paramecium aurelia after homologus antiserum treatment during autogamy and conjugation. J. Protozool. (Suppl.) 6; 25, 1959.

13. Kawata, S., Sasaki, K. and Minami, S.: Component analysis of spatial and spectral patterns in multispectral image. I. Basis. J. Opt. Soc. Am. 4; 2101-2106, 1987.

14. Nakae, Y. and Stoward, P. J.: Initial rate reaction kinetics of succinate dehydrogenase in mouse liver studied with a real-time image analyzer system. Histochemistry 98; 7-12, 1992.

15. Van Noorden, C. J. F. and Vogels, I. M. C.: Polyvinyl alcohol and other tissue protectants in enzyme histochemistry: A consumer's guide. Histochem. J. 21; 373-379, 1989.

16. Van Noorden, C. J. F. and Butcher, R. G.: Quantitative histochemistry. In "Histochemistry", vol. 3, ed. by P. J. Stoward and A. G. E. Pearse, 4th ed., Churchhill Livingstone, London, 1991, pp. 367-377.

17. Van Noorden, C. J. F. and Frederiks, W. M.: Unit of comparison of biochemical and quantitative histochemical data. In "Enzyme Histochemistry. A Laboratory Manual of Current Methods", Royal microscopical Society, Microscopy handbook 26, Oxford University Press Inc., New York, 1992, pp. 26-29.

18. Wichterman, R.: The biology of Paramecium, 2nd ed., Pleum Press, New York, 1986, pp. 80-83.

19. Wichterman, R.: The biology of Paramecium, 2nd ed., Pleum Press. New York, 1986, pp. 120-121. 